\title{
Approximate Analytical Description of the Projectile Motion with a Quadratic Drag Force
}

\author{
By Peter Chudinov *
}

\begin{abstract}
In this paper, the problem of the motion of a projectile thrown at an angle to the horizon is studied. With zero air drag force, the analytic solution is well known. The trajectory of the projectile is a parabola. In situations of practical interest, such as throwing a ball with the occurrence of the impact of the medium the quadratic resistance law is usually used. In that case the problem probably does not have an exact analytic solution and therefore in most scientific publications it is solved numerically. Analytic approaches to the solution of the problem are not sufficiently advanced. Meanwhile, analytical solutions are very convenient for a straightforward adaptation to applied problems and are especially useful for a qualitative analysis. That is why the description of the projectile motion with a simple approximate analytical formula under the quadratic air resistance represents great methodological interest. Lately these formulas have been obtained. These formulas allow us to obtain a complete analytical description of the problem. This description includes analytical formulas for determining the basic eight parameters of projectile motion. Analytical formulas have been derived for the six basic functional dependences of the problem, including the trajectory equation in Cartesian coordinates. Also this description includes the determination of the optimum throwing angle and maximum range of the motion. In the absence of air resistance, all these relations turn into well-known formulas of the theory of the parabolic motion of the projectile. The proposed analytical solution differs from other solutions by simplicity of formulas, ease of use and high accuracy (relative error is about 1-2 \%). The motion of a baseball is presented as an example. The proposed formulas make it possible to carry out an analytical investigation of the motion of a projectile in a medium with resistance in the way it is done in the case of no drag.
\end{abstract}

\section{Introduction}

The problem of the motion of a point mass (projectile) thrown at an angle to the horizon in midair has a long history. It is one of the great classical problems that started the dynamics as a science. The number of works devoted

*Associated Professor, Perm State Agricultural Academy, Russian Federation. 
to this task is immense. It represents a constituent of many introductory courses of physics. With no drag, the analytic solution of this problem is well known. The trajectory of the point mass is a parabola. In this paper, an analytic approach is used for the investigation of the projectile motion in a medium with quadratic resistance. The object of the present work is to give simple formulas for the construction of the projectile trajectories under the motion with quadratic air resistance. These formulas are available even for first-year undergraduates.

The problem of the motion of a projectile in midair has aroused interest of authors (Cohen et al., 2014; Kantrowitz \& Neumann, 2013; Borghi, 2013). For the construction of the analytical solutions various methods are used - both the traditional approaches (Benacka, 2010; Vial, 2007; Parker, 1977; Erlichson, 1983; Tan, Frick \& Castillo, 1987)], and the modern methods (Yabushita, Yamashita \& Tsuboi, 2007). All proposed approximate analytical solutions are rather complicated and inconvenient for educational purposes. This is why the description of the projectile motion by means of a simple approximate analytical formula under the quadratic air resistance is of great methodological and educational importance. In (Chudinov, 2002; 2004; 2013) comparatively simple approximate analytical formulas have been obtained to study the motion of the projectile in a medium with a quadratic drag force. In this article, these formulas are used to solve the classical problem of maximizing the projectile distance. From now on, the term "point mass" means the center of mass of a smooth spherical object of finite radius $r$ and cross-sectional area $S=\pi r^{2}$. The conditions of applicability of the quadratic resistance law are deemed to be fulfilled, i.e. Reynolds number Re lies within $1 \times 10^{3}<\operatorname{Re}<2 \times 10^{5}$. These values correspond to the projectile motion velocity, lying in the range between $0.25 \mathrm{~m} / \mathrm{s}$ and $53 \mathrm{~m} / \mathrm{s}$.

\section{Equations of Point Mass Motion and Analytical Formulas for Basic Parameters}

Suppose that the force of gravity affects the point mass together with the force of air resistance $\boldsymbol{R}$ (see Fig. 1). Air resistance force is proportional to the square of the velocity of the point mass and is directed opposite the velocity vector. For the convenience of further calculations, the drag force will be written as $R=m g k V^{2}$. Here $m$ is the mass of the projectile, $g$ is the acceleration due to gravity, $k$ is the proportionality factor. Vector equation of the motion of the point mass has the form

$$
m \boldsymbol{w}=m \boldsymbol{g}+\boldsymbol{R},
$$

where $\boldsymbol{w}$ - acceleration vector of the point mass. Differential equations of the motion, commonly used in ballistics, are as follows (Okunev, 1943) 
$\frac{d V}{d t}=-g \sin \theta-g k V^{2}, \frac{d \theta}{d t}=-\frac{g \cos \theta}{V}, \frac{d x}{d t}=V \cos \theta, \frac{d y}{d t}=V \sin \theta$

Here $V$ is the velocity of the point mass, $\theta$ is the angle between the tangent to the trajectory of the point mass and the horizontal, $x, y$ are the Cartesian coordinates of the point mass, $k$ is

$$
k=\frac{\rho_{a} c_{d} S}{2 m g}=\frac{1}{V_{\text {term }}^{2}}=\text { const },
$$

$\rho_{a}$ is the air density, $c_{d}$ is the drag factor for a sphere, $S$ is the cross-section area of the object, and $V_{\text {term }}$ is the terminal velocity. The first two equations of the system (1) represent the projections of the vector equation of motion on the tangent and principal normal to the trajectory; the other two are kinematic relations connecting the projections of the velocity vector point mass on the axis $x, y$ with derivatives of the coordinates.

Figure 1. Basic Motion Parameters

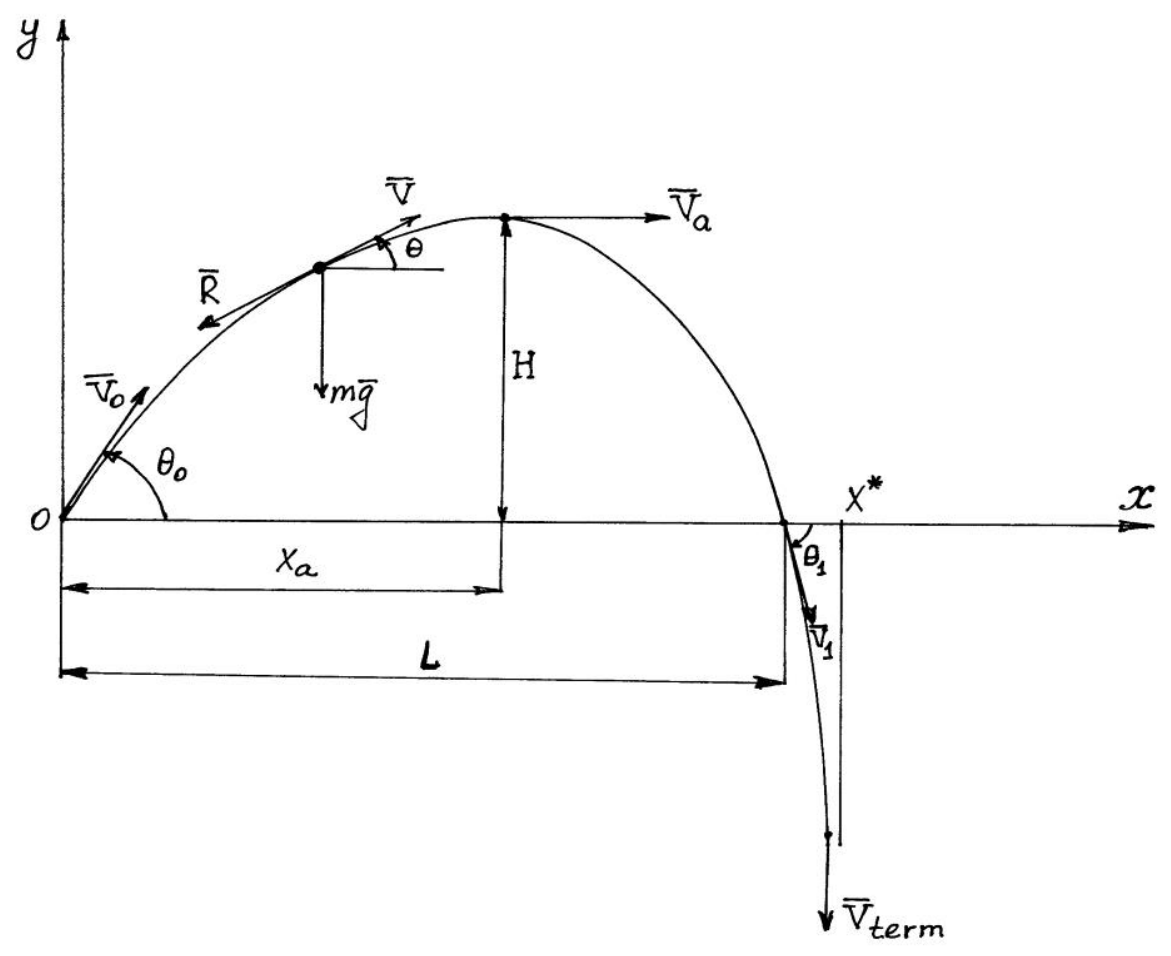

The well-known solution of system (1) consists of an explicit analytical dependence of the velocity on the slope angle of the trajectory and three quadratures

$$
V(\theta)=\frac{V_{0} \cos \theta_{0}}{\cos \theta \sqrt{1+k V_{0}^{2} \cos ^{2} \theta_{0}\left(f\left(\theta_{0}\right)-f(\theta)\right)}},
$$


Vol. 1, No. 2 Chudinov: Approximate Analytical Description of the Projectile Motion...

$$
\begin{gathered}
f(\theta)=\frac{\sin \theta}{\cos ^{2} \theta}+\ln \operatorname{tg}\left(\frac{\theta}{2}+\frac{\pi}{4}\right) \\
t=t_{0}-\frac{1}{g} \int_{\theta_{0}}^{\theta} \frac{V}{\cos \theta} d \theta, x=x_{0}-\frac{1}{g} \int_{\theta_{0}}^{\theta} V^{2} d \theta, y=y_{0}-\frac{1}{g} \int_{\theta_{0}}^{\theta} V^{2} \operatorname{tg} \theta d \theta
\end{gathered}
$$

Here $V_{0}$ and $\theta_{0}$ are the initial values of the velocity and the slope of the trajectory respectively, $t_{0}$ is the initial value of the time, $x_{0}, y_{0}$ are the initial values of the coordinates of the point mass (usually accepted $t_{0}=x_{0}=y_{0}=0$ ). The derivation of the formulas (2) is shown in the well-known monograph (Timoshenko \& Young, 1948). The integrals on the right-hand sides of formulas (3) cannot be expressed in terms of elementary functions. Hence, to determine the variables $t, x$ and $y$ we must either integrate system (1) numerically or evaluate the definite integrals (3).

Comparatively simple approximate analytical formulas for the main parameters of motion of the projectile are derived in (Chudinov, 2002, 2004). The four parameters correspond to the top of the trajectory, four - to the point of drop. We will give a complete summary of the formulas for the maximum height of ascent of the point mass $H$, motion time $T$, the velocity at the trajectory apex $V_{a}=V(0)$, flight range $L$, the time of ascent $t_{a}$, the abscissa of the trajectory apex $x_{a}$, impact angle with respect to the horizontal $\theta_{1}$ and the final velocity $V_{1}$ (see Fig. 1). These formulas are summarized in the right column of Table 1 . In the left column of this Table 1 similar formulas of the parabolic theory are presented for comparison.

Table 1. Analytical Formulas for the Main Parameters

\begin{tabular}{|c|c|}
\hline No drag $(R=0)$ & Quadratic drag force $\left(R=m g k V^{2}\right)$ \\
\hline$H=\frac{V_{0}^{2} \sin ^{2} \theta_{0}}{2 g}$ & $H=\frac{V_{0}^{2} \sin ^{2} \theta_{0}}{g\left(2+k V_{0}^{2} \sin \theta_{0}\right)}$ \\
\hline$T=2 \frac{V_{0} \sin \theta_{0}}{g}=2 \sqrt{\frac{2 H}{g}}$ & $V_{a}=\frac{T=2 \sqrt{\frac{2 H}{g}}}{\sqrt{1+k V_{0}^{2}\left(\sin \theta_{0}+\cos ^{2} \theta_{0} \cdot \ln \tan \left(\frac{\theta_{0}}{2}+\frac{\pi}{4}\right)\right)}}$ \\
\hline$V_{a}=V_{0} \cos \theta_{0}$ & \begin{tabular}{c}
$L=V_{a} T$ \\
\hline$L=\frac{1}{g} V_{0}^{2} \sin 2 \theta_{0}=V_{a} T$
\end{tabular} \\
\hline$t_{a}=\frac{V_{0} \sin \theta_{0}}{g}=\frac{T}{2}$ & $t_{a}=\frac{T-k H V_{a}}{2}$ \\
\hline
\end{tabular}




\begin{tabular}{|c|c|}
$x_{a}=\frac{L}{2}=\sqrt{L H \cot \theta_{0}}$ & $x_{a}=\sqrt{L H \cot \theta_{0}}$ \\
\hline$\theta_{1}=-\theta_{0}=$ & \\
$=-\arctan \left[\frac{L H}{\left(L-x_{a}\right)^{2}}\right]$ & $\theta_{1}=-\arctan \left[\frac{L H}{\left(L-x_{a}\right)^{2}}\right]$ \\
\hline$V_{1}=V_{0}$ & $V_{1}=V\left(\theta_{1}\right)$ \\
\hline
\end{tabular}

With zero drag $(k=0)$, these formulas go over into the respective formulas of the point mass parabolic motion theory. All motion characteristics described by these formulas are functions of initial conditions of throwing $V_{0}, \theta_{0}$.

Proposed formulas have a bounded region of application. We introduce the notation $p=k V_{0}^{2}$. The dimensionless parameter $p$ has the following physical meaning - it is the ratio of air resistance to the weight of the projectile at the beginning of the movement. The main characteristics of the motion $H, T, V_{a}$, $L, x_{a}$ have accuracy to within $2-3 \%$ for values of the launch angle, for initial velocity and for the parameter $p$ from ranges

$$
0^{\circ} \leq \theta_{0} \leq 70^{\circ}, \quad 0 \leq V_{0} \leq 50 \mathrm{~m} / \mathrm{s}, \quad 0 \leq p \leq 1.5 \text {. }
$$

For a baseball the typical values of the drag force coefficient $k$ are about $0.0005 \div 0.0006 \mathrm{~s}^{2} / \mathrm{m}^{2}$, maximal initial velocity is about $50 \mathrm{~m} / \mathrm{s}$ (Cohen et al., 2014; Tan et al., 1987). Therefore the proposed formulas are suitable for the qualitative and quantitative description of the motion of the baseball and other similar objects.

These formulas, in turn, make it possible to obtain a simple analytical formula for the main functional relationship of the problem $y(x)$ (Chudinov, 2002). In the absence of air resistance, the trajectory of a point mass is a parabola. The equation of the trajectory can be written in two forms. It can be written in terms of the initial conditions of throwing $V_{0}, \theta_{0}$ (first form). It can also be written in terms of the motion parameters $H, L, x_{a}$ (second form)

$$
y(x)=x \cdot \tan \theta_{0}-\frac{g x^{2}}{2 V_{0}^{2} \cos ^{2} \theta_{0}}=\frac{H x(L-x)}{x_{a}^{2}}
$$

The trajectory is symmetric with respect to the maximum. When the point mass is under a drag force, the trajectory becomes asymmetrical. The top of the trajectory is shifted towards the point of incidence. In addition, a vertical asymptote appears near the trajectory. Taking these circumstances into account, the function $y(x)$ may be constructed using parameters $H, L, x_{a}$ as (Chudinov, 2002) 
Vol. 1, No. 2 Chudinov: Approximate Analytical Description of the Projectile Motion...

$$
y(x)=\frac{H x(L-x)}{x_{a}^{2}+\left(L-2 x_{a}\right) x}
$$

The constructed dependence $y(x)$ provides the shift of the apex of the trajectory to the right and has a vertical asymptote. In the case of no drag $L=$ $2 x_{a}$ and formula (5) goes over to formula (4). We note the remarkable property of formula (5). We insert the exact values of the parameters $L, H, x_{a}$, obtained by numerical integration of system (1), into formula (5). Then the numerical trajectory and the analytical trajectory constructed by means of formula (5) are identical. This means that formula (5) approximates absolutely precisely the projectile's trajectory which is numerically constructed using system (1) at any values of the initial conditions $V_{0}, \theta_{0}$.

The some transformation of the proposed formulas (Chudinov, 2013) makes it possible to improve the accuracy of calculating the main parameters. Now it is possible to construct the trajectory in the entire range of launch angles and at values of the initial velocity and the parameter $p$

$$
0^{\circ} \leq \theta_{0} \leq 90^{\circ}, \quad 0 \leq V_{0} \leq 80 \mathrm{~m} / \mathrm{s}, \quad 0 \leq p \leq 4 \text {. }
$$

As an example of the use of the proposed formulas from the Table 1 and of formula (5) we calculated the motion of a baseball with the following initial conditions

$$
V_{0}=40 \mathrm{~m} / \mathrm{s}, \theta_{0}=45^{\circ}, k=0.000625 \mathrm{~s}^{2} / \mathrm{m}^{2}, g=9.81 \mathrm{~m} / \mathrm{s}^{2} .
$$

Table 2. Comparison of Numerical and Analytical Calculations

\begin{tabular}{|c|c|c|c|}
\hline Parameter & Analytical value & Numerical value & Error (\%) \\
\hline$H(\mathrm{~m})$ & 30.1 & 29.8 & 1.1 \\
\hline$T(\mathrm{~s})$ & 4.96 & 4.91 & 1.0 \\
\hline$V_{a}(\mathrm{~m} / \mathrm{s})$ & 19.3 & 19.3 & 0 \\
\hline$L(\mathrm{~m})$ & 95.7 & 96.1 & -0.4 \\
\hline$t_{a}(\mathrm{~s})$ & 2.30 & 2.31 & -0.4 \\
\hline$x_{a}(\mathrm{~m})$ & 53.7 & 53.0 & 1.3 \\
\hline$\theta_{1}($ degree $)$ & $-58.6^{\circ}$ & $-57.3^{\circ}$ & 2.2 \\
\hline$V_{1}(\mathrm{~m} / \mathrm{s})$ & 26.0 & 25.5 & 2.0 \\
\hline
\end{tabular}

The results of calculations are recorded in Table 2 and are shown in Figure 2. The second column contains the values calculated by present formulas from the Table 1. The third column shows the values of parameters obtained by numerical integration of the motion equations (1) by the fourth-order RungeKutta method. The deviations from the exact values of parameters are shown in the fourth column of the Table 2. Tabulated data show that the values of basic parameters of the projectile motion (flight range $L$, motion time $T$, height $H$ ) calculated by analytical formulas differ from the exact values no more than $1 \%$. 
An approximate trajectory (5) is constructed with the help of parameters $H, L, x_{a}$. It is shown in Figure 2 (dotted line). The thick solid line in Figure 2 is obtained by numerical integration of system (1) with the aid of the 4-th order Runge-Kutta method. As can be seen from Figure 2, the analytical solution (formula (5)) and a numerical solution are almost the same. The dashed line in Figure 2 is constructed in the absence of air resistance.

Figure 2. The Graph of the Trajectory $y=y(x)$

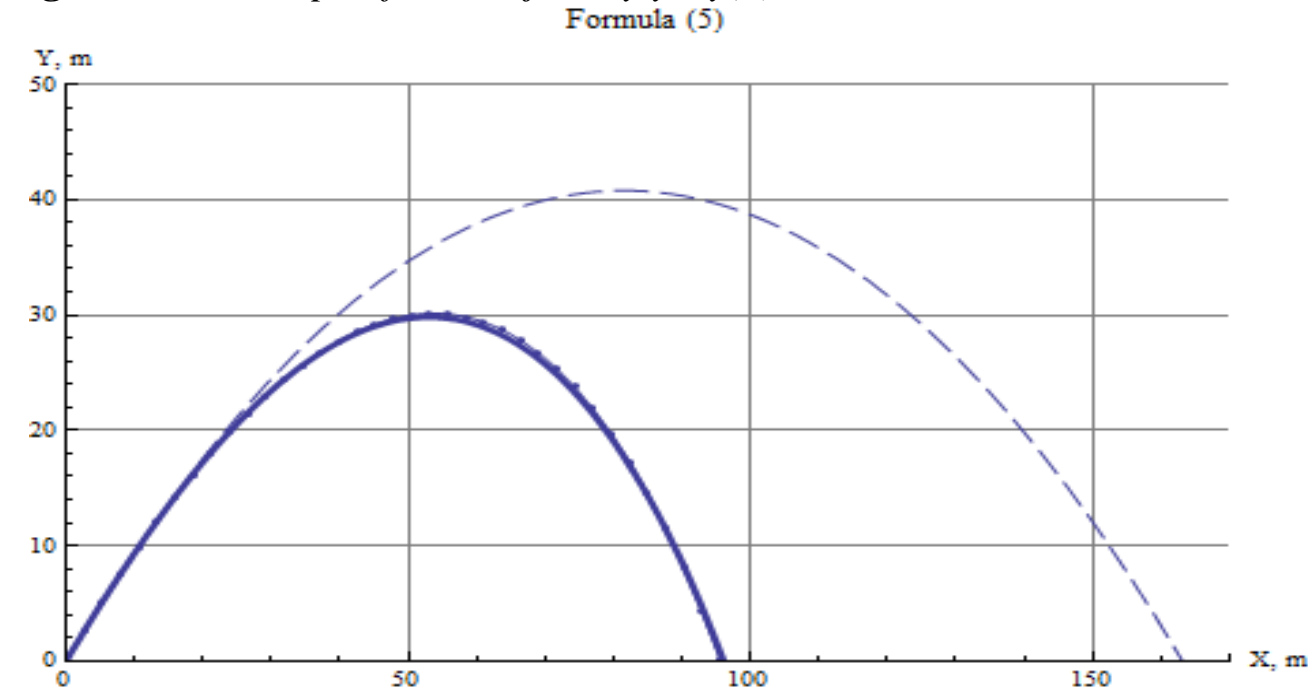

One of the most important aspects of the projectile motion problem is determination of an optimum angle of throwing of a point mass which provides the maximum range. Let us make use of formulas from the Table 1 and (5) to solve the problem of optimization of motion parameters.

\section{Determination of an Optimum Throwing Angle and Maximum Range}

Let the point of incidence $\mathrm{A}$ be on a horizontal straight line defined by the equation $y=y_{1}=$ const (see Fig.3).

Figure 3. Statement of the Optimization Problem

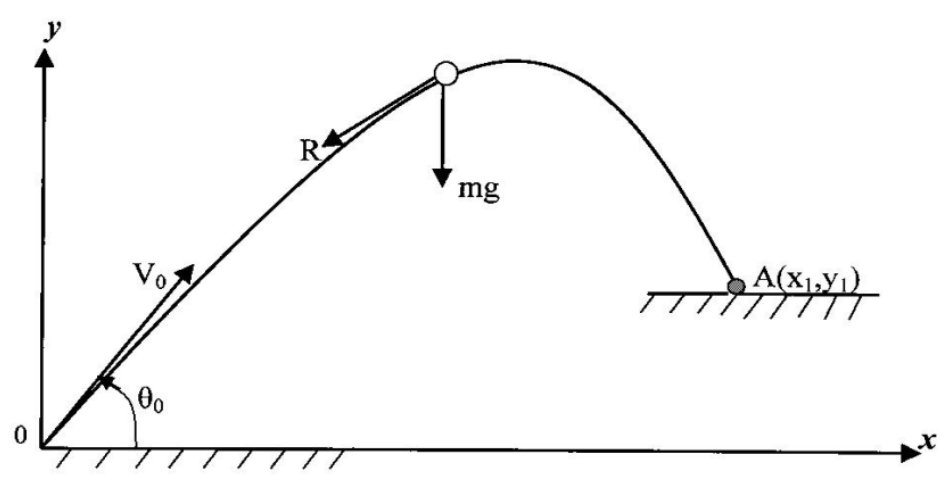


To solve the problem, let us make use of the point mass trajectory equation (5). In this equation, the motion characteristics $H, L, x_{a}$ are functions of initial conditions of throwing, $V_{0}, \theta_{0}$. They are defined by formulas from the Table 1 . Let us substitute $y_{1}$ in the left-hand part of equation (5) and solve it for variable $x$. Thus, we shall get the flight range formula for the case under consideration

$$
x\left(V_{0}, \theta_{0}, y_{1}\right)=\delta+\sqrt{\delta^{2}-L y_{1} \cot \theta_{0}},
$$

where $\delta=\frac{L}{2}+\frac{y_{1}}{H}\left(x_{a}-\frac{L}{2}\right)$. For the given values of the $V_{0}, y_{1}$ parameters, range $x$ is the function of $\theta_{0}$, the angle of throwing. To find the optimum angle of throwing $\theta_{0}^{\text {opt }}$ and the maximum range $x_{\max }$ gained with the given $V_{0}, y_{1}$ values, it is sufficient to construct a graph of this function. Coordinates of the maximum point of the function will define values of $\theta_{0}^{\text {opt }}$ and $x_{\max }$.

As an example, let us analyze the motion of a baseball with the drag factor $k=0.000625 \mathrm{~s}^{2} / \mathrm{m}^{2}$ (Cohen et al., 2014). Other parameters of the motion are given by the following values:

$$
g=9.81 \mathrm{~m} / \mathrm{s}^{2}, \quad V_{0}=40 \mathrm{~m} / \mathrm{s}, \quad y_{1}=0, \pm 20 \mathrm{~m} .
$$

Computation results are presented in Figure 4 and Table 3. The graphs of the $x\left(\theta_{0}\right)$ functions (6) for $y_{1}=0, \pm 20 \mathrm{~m}$ are given in Figure 4. In Figure 4, points $\mathrm{A}, \mathrm{B}, \mathrm{C}$ are the points of the maximum of the functions $x\left(\theta_{0}\right)$ for values $20,0,-20$ of the parameter $y_{1}$ accordingly. Numerical values of the parameters $\theta_{0}^{\text {opt }}$ and $x_{\text {max }}$ in column 5 of the Table 3 were obtained by integrating the system (1).

Figure 4. The Graphs of the $x=x\left(\theta_{0}\right)$ Functions

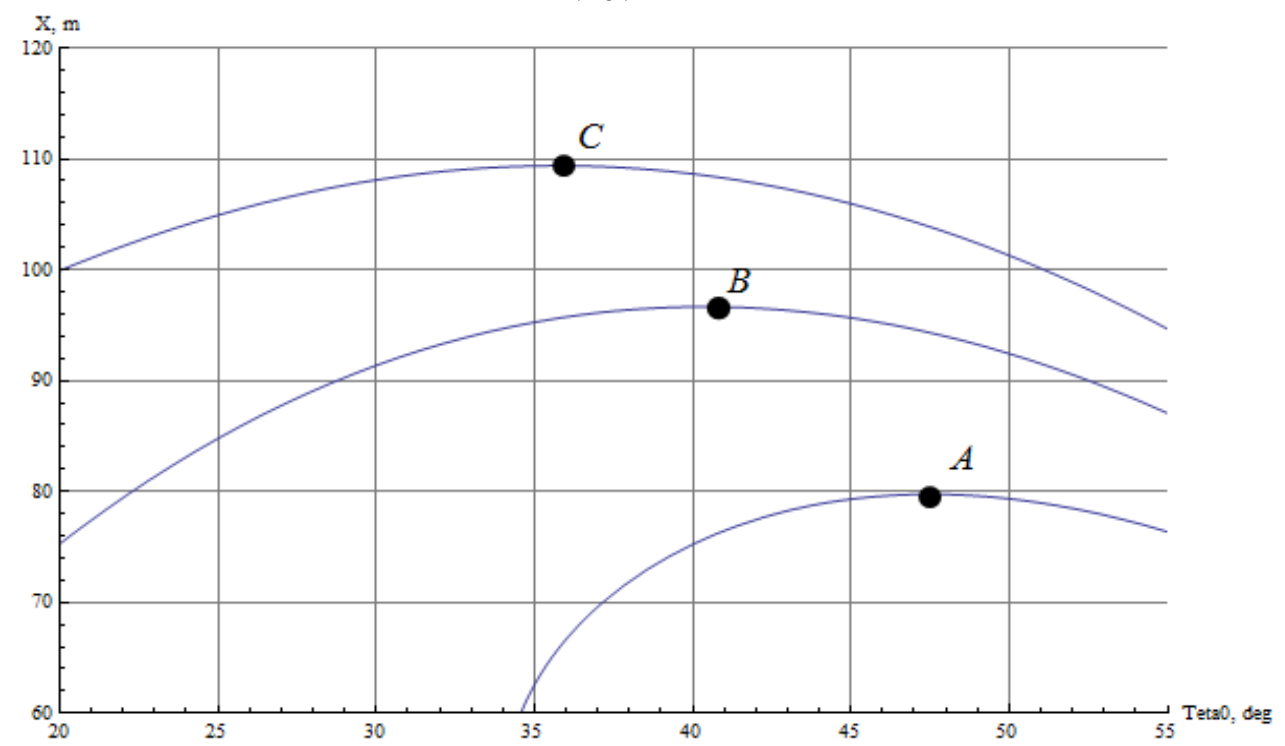


Table 3. Optimum Trajectory Parameters

\begin{tabular}{|c|c|c|c|c|c|}
\hline $\begin{array}{l}\text { Value } \\
y_{1} \\
(\mathrm{~m})\end{array}$ & $\begin{array}{l}\text { The point of the } \\
\text { maximum of the } \\
\text { function } x\left(\theta_{0}\right)\end{array}$ & Parameter & $\begin{array}{l}\text { Analytic } \\
\text { value from } \\
\text { Figure } 4\end{array}$ & $\begin{array}{c}\text { Numeric } \\
\text { value }\end{array}$ & $\begin{array}{l}\text { Error } \\
(\%)\end{array}$ \\
\hline 20 & Point A & $\begin{array}{c}\theta_{0}^{\text {opt }}(\text { degree }) \\
x_{\max }(\mathrm{m})\end{array}$ & $\begin{array}{c}47.5^{\circ} \\
79.7\end{array}$ & $\begin{array}{l}47.8^{\circ} \\
79.6\end{array}$ & $\begin{array}{c}-0.6 \\
0.1\end{array}$ \\
\hline 0 & Point B & $\begin{array}{c}\theta_{0}^{\text {opt }}(\text { degree }) \\
x_{\max }(\mathrm{m})\end{array}$ & $\begin{array}{l}40.8^{\circ} \\
96.6\end{array}$ & $\begin{array}{l}40.8^{\circ} \\
96.8\end{array}$ & $\begin{array}{c}0 \\
-0.2\end{array}$ \\
\hline-20 & Point $\mathrm{C}$ & $\begin{array}{c}\theta_{0}^{o p t} \\
\text { (degree) } x_{\text {max }} \\
(\mathrm{m})\end{array}$ & $\begin{array}{l}35.8^{\circ} \\
109.8\end{array}$ & $\begin{array}{l}35.8^{\circ} \\
110.1\end{array}$ & $\begin{array}{c}0 \\
-0.3\end{array}$ \\
\hline
\end{tabular}

Tabulated data show that formulas from the Table 1 and (6) make it possible not only to simplify the considerably solution of the problem but also provide a high accuracy of computations. The $\theta_{0}^{\text {opt }}$ angle is determined accurately and the range is determined with an error of $\pm 0.6 \%$.

\section{Summary}

The proposed approach based on the use of analytic formulas makes it possible to simplify significantly a qualitative analysis of the motion of a projectile with the air drag taken into account. All basic parameters of motion and various problems of optimization are described by simple analytical formulas containing elementary functions. Moreover, numerical values of the sought variables are determined with an acceptable accuracy. It can be implemented even on a standard calculator. Lately some authors (Warburton \& Wang, 2004; Stewart, 2005; Hu et al., 2012) have used the Lambert W function to study the projectile motion with resistance. But this relatively "new" function is not available on a calculator. Special algorithms are required to compute this function. Thus, proposed formulas make it possible to study projectile motion with quadratic drag force even for first-year undergraduates. In conclusion, we hope that efforts to obtain an analytical solution to this problem will be continued and will achieve new exact and efficient solutions.

\section{Acknowledgements}

The author would like to thank Dr. D.V. Zitta and Dr. A.V. Fainshtein for the valuable advice for this paper. 
Vol. 1, No. 2 Chudinov: Approximate Analytical Description of the Projectile Motion...

\section{References}

Baće, M., Ilijić, S., Narancić, Z., Bistricić, L. (2002). The envelope of projectile trajectories. European Journal of Physics. 23, 637-642.

Benacka, J. (2010). Solution to projectile motion with quadratic drag and graphing the trajectory in spreadsheets. International Journal of Mathematical Education in Science and Technology. 41, 373-378.

Borghi, R. (2013). Trajectory of a body in a resistance medium: an elementary derivation. European Journal of Physics. 34, 359-370.

Chudinov, P.S. (2002). The motion of a heavy particle in a medium with quadratic drag force. International Journal of Nonlinear Sciences and Numerical Simulation. 3, 121-129.

Chudinov, P.S. (2004). An optimal angle of launching a point mass in a medium with quadratic drag force. In Proceedings of 21-th International Ballistics Symposium (Adelaide, South Australia, April 19 - 23, 2004, Vol. 1, 511-517).

Chudinov, P.S. (2004). Analytical investigation of point mass motion in midair. European Journal of Physics. 25, 73-79.

Chudinov, P.S. (2013). Extension of application field of analytical formulas for the computation of projectile motion in midair. Revista Brasileira de Ensino de Fisica. 35(1), 1310-1314.

Cohen, C., Darbois-Texier, B., Dupeux, G., Brunel, E., Quere, D., Clanet, C. (2014). The aerodynamic wall. In Proceedings of the Royal Society A. 470, 20130497. DOI=http://dx.doi.org/10.1098/rspa.2013.0497.

Erlichson, H. (1983). Maximum projectile range with drag and lift, with particular application to golf. American Journal of Physics. 51, 357-362.

Hu, H., Zhao, Y.P., Guo, Y.J., Zheng, M.Y. (2012). Analysis of linear resisted projectile motion using the Lambert W function. Acta Mechanica. 223, 441-447.

Kantrowitz, R., Neumann, M.M. (2013).Some real analysis behind optimization of projectile motion. Mediterranean Journal of Mathematics. DOI:10.1007/s00009013-0379-5.

Okunev, B.N. (1943). Ballistics. Moscow: Voyenizdat.

Parker, G. W. (1977). Projectile motion with air resistance quadratic in the speed. American Journal of Physics. 45, 606-610.

Stewart, S.M. (2005). Linear resisted projectile motion and the Lambert W function. American Journal of Physics. 73, 199-199.

Tan, A., Frick, C.H., Castillo, O. (1987). The fly ball trajectory: an older approach revisited. American Journal of Physics. 55, 37-40.

Timoshenko, S., Young, D.H. (1948). Advanced Dynamics. New York: McGrow-Hill Book Company.

Vial, A. (2007). Horizontal distance travelled by a mobile experiencing a quadratic drag force: normalized distance and parameterization. European Journal of Physics. 28, 657-663.

Warburton, R.D.H., Wang, J. (2004). Analysis of asymptotic projectile motion with air resistance using the Lambert W function. American Journal of Physics. 72, 1404-1407.

Yabushita, K., Yamashita, M., Tsuboi, K. (2007). An analytic solution of projectile motion with the quadratic resistance law using the homotopy analysis method. Journal of Physics A: Mathematical and Theoretical. 40, 8403-8416. 